\title{
Control and femtosecond time-resolved imaging of torsion in a chiral molecule
}

\author{
Jonas L. Hansen, ${ }^{1}$ Jens H. Nielsen, ${ }^{2}$ Christian Bruun Madsen, ${ }^{3}$ \\ Anders Thyboe Lindhardt, ${ }^{1,4}$ Mikael P. Johansson, ${ }^{5}$ Troels Skrydstrup, ${ }^{1,4}$ \\ Lars Bojer Madsen, ${ }^{6}$ and Henrik Stapelfeldt ${ }^{1,4}$ \\ ${ }_{1}^{1}$ Interdisciplinary Nanoscience Center (iNANO), Aarhus University, DK-8000 Aarhus C, Denmark \\ ${ }^{2}$ Department of Physics and Astronomy, Aarhus University, DK-8000 Aarhus C, Denmark \\ ${ }^{3}$ J. R. Macdonald Laboratory, Kansas State University, Manhattan, Kansas 66506, USA \\ ${ }^{4}$ Department of Chemistry, Aarhus University, DK-8000 Aarhus C, Denmark \\ ${ }^{5}$ Institut de Química Computacional and Departament de Química, Universitat de Girona, \\ Campus Montilivi, ES-17071 Girona, Spain \\ ${ }^{6}$ Lundbeck Foundation Theoretical Center for Quantum System Research, Department of Physics \\ and Astronomy, Aarhus University, DK-8000 Aarhus C, Denmark
}

(Received 26 March 2012; accepted 3 May 2012; published online 29 May 2012)

\begin{abstract}
We study how the combination of long and short laser pulses can be used to induce torsion in an axially chiral biphenyl derivative (3,5-difluoro-3',5'-dibromo-4'-cyanobiphenyl). A long, with respect to the molecular rotational periods, elliptically polarized laser pulse produces $3 \mathrm{D}$ alignment of the molecules, and a linearly polarized short pulse initiates torsion about the stereogenic axis. The torsional motion is monitored in real-time by measuring the dihedral angle using femtosecond time-resolved Coulomb explosion imaging. Within the first 4 picoseconds (ps), torsion occurs with a period of $1.25 \mathrm{ps}$ and an amplitude of $3^{\circ}$ in excellent agreement with theoretical calculations. At larger times, the quantum states of the molecules describing the torsional motion dephase and an almost isotropic distribution of the dihedral angle is measured. We demonstrate an original application of covariance analysis of two-dimensional ion images to reveal strong correlations between specific ejected ionic fragments from Coulomb explosion. This technique strengthens our interpretation of the experimental data. (C) 2012 American Institute of Physics. [http://dx.doi.org/10.1063/1.4719816]
\end{abstract}

\section{INTRODUCTION}

The control of molecules and chemical reactions with lasers is one of the main goals of femtochemistry. ${ }^{1}$ One particular aspect of such studies is the use of laser pulses to control the transition from one enantiomer of a chiral molecule to its mirror form. This topic has been the subject of a large number of theoretical studies and these efforts are motivated by the intriguing prospect of light-induced deracemization, ${ }^{2-8}$ i.e., creation of enantiomeric excess. Axially chiral molecules have attracted special interest because the potential barrier separating an enantiomeric pair is often low so that lasers at moderate intensities may be applied to facilitate the efficient crossing of the barrier without fragmenting the molecule. For axially chiral molecules, the stereogenic element is an axis (connecting two atoms) rather than a point (a single atom) and the reaction path separating the two enantiomers corresponds to torsion around the stereogenic axis. ${ }^{9}$

The first theoretical light-based scheme, which successfully showed the production of enanotiomeric excess $(50.0001 \%: 49.9999 \%)$, involved circularly polarized continuous wave monochromatic light. ${ }^{2}$ However, a much higher conversion rate is needed if the deracemization is to be confirmed experimentally — not to mention to have practical applications. To this end, studies showed that almost perfect deracemization could be achieved by exposing phosphinothioic acid $\left(\mathrm{H}_{2} \mathrm{OPSH}\right)$ to picosecond terahertz laser pulses. ${ }^{3}$ In these studies, the key was to realize that changes in the torsional coordinate were associated with a shift in the permanent dipole moment, a feature that was exploited by using polarization shaped laser pulses to transfer a racemate into the desired enantiomeric form. Since this discovery, several different approaches have been proposed to control torsional motion in axially chiral molecules. These approaches include the use of coherent control or phase controlled pulses ${ }^{10}$ to perform, e.g., laser distillation, ${ }^{4}$ where the excitation of one of the enantiomers can be enhanced by coupling the ground state to a superposition of two excited states with opposite parities, or by cyclic population transfer that relies on quantum interference in an effective three-level-system. ${ }^{5}$ Alternatively, the deracemization can be achieved by exploiting that the direction of the electronic transition moment vector to an excited state is different for the two enantiomers. One way to exploit this directionality is a pump-dump scheme, ${ }^{6}$ where the population is gradually transferred from one enantiomer to the other. Another method would be asymmetric excitation, where the excited state is repulsive and leads to dissociation thereby removing this enantiomeric form from the ensemble. ${ }^{7}$ A common feature for most of these schemes is that they either require or benefit from pre-aligned and oriented molecular targets. ${ }^{8}$

Since alignment and orientation are generally required in the enantiomer-control-schemes, a more straightforward, and thereby experimentally feasible approach, would be to rely on 
alignment concepts alone to control the torsion. Recently, this has been the focus of several studies. These studies fall into two regimes: (i) the adiabatic regime, where the pump pulse that induces torsion is long compared to the time scale of torsional dynamics ${ }^{11-13}$ and (ii) torsion induced by a short nonadiabatic pump pulse. ${ }^{13-15}$ For those axially chiral molecules where the stereogenic axis and the most polarizable axis coincide, it was suggested that torsion could be controlled adiabatically by a single elliptically polarized nanosecond (ns) laser pulse provided that the torsional barrier is sufficiently low. This pulse potentially serves to both induce 3D alignment and torsional motion by interaction of the minor axis of the polarization ellipse with the polarizability components of the two moieties forcing them into coplanarity. ${ }^{11,13}$ The feasibility of this approach has recently been disputed due to the coupling between overall rotation and torsional motion leading to a breakdown of torsional alignment. ${ }^{12}$

So far, only very few experimental papers have investigated for the possibility to control and do real-time monitoring of the torsion in axially chiral molecules. In Refs. 14 and 15, the stereogenic axis of a 3,5-difluoro- $3^{\prime}, 5^{\prime}$-dibromobiphenyl molecule was held fixed-in-space by 1-dimensional (1D) adiabatic alignment with a linearly polarized $9 \mathrm{~ns}$ long laser pulse. In equilibrium, the angle between the F-phenyl plane and the Br-phenyl plane is $\pm 39^{\circ}$. Upon irradiation with a 700 femtosecond (fs) long, intense (but non-fragmenting), nonresonant pump pulse, torsion was induced, accompanied by overall rotation around the fixed stereogenic axis. The torsional and rotational dynamics, measured by inducing Coulomb explosion with a short intense delayed probe pulse and recording of the emission direction of recoiling $\mathrm{Br}^{+}$ and $\mathrm{F}^{+}$ions through ion imaging, showed that the amplitude and period of torsion was $0.6^{\circ}$ and 1 picosecond (ps). While the period was in qualitative agreement with the theoretical model prediction of $1.2 \mathrm{ps}$, the amplitude predicted by theory was larger, $2.45^{\circ}$. The theory rationalized that stimulated Raman transitions, driven by the pump pulse, creates a wave packet of torsional eigenstates in the electronic ground state leading to the torsion observed. It is one of the aims of the present work to resolve the discrepancy between theory and experiment.

The principle of the current work is similar to the previous work, ${ }^{14,15}$ but three key experimental factors were changed to achieve much better resolved peaks in the experimental ion images, which allows for the tracing of the torsional motion with unprecedented precision and, therefore, a quantitative rather than a qualitative comparison to the calculated results. The three decisive factors are: (1) A chemically much purer sample resulting in higher contrast ion images. (2) The molecules were $3 \mathrm{D}$ aligned ${ }^{16,17}$ prior to the kick and the probe pulses, i.e., the entire molecule was fixed-inspace not just the stereogenic axis (1D alignment). This implies that the torsional motion stands out much more distinctly in the ion images. (3) The molecule employed here, 3,5difluoro- $3^{\prime}, 5^{\prime}$-dibromo-4'-cyanobiphenyl, is almost identical to the molecule studied in Refs. 14 and 15 except that it has a nitrile group attached to the end of the Br-phenyl. This gives the current molecule a dipole moment of 4.4 Debye $^{18,35-40}$ compared to 0.2 Debye $^{18}$ for the molecule used previously.
The large permanent dipole moment of the molecules made it possible to use an electrostatic deflector to spatially separate molecules according to their rotational quantum state. ${ }^{19,20}$ Performing the separation prior to the interaction with the laser pulses allowed us to focus the laser pulses onto only the molecules with low rotational energy, improving the degree of prealignment. ${ }^{19,20}$

The paper is organized as follows. In Sec. II, the experimental setup is described. In Sec. III, the results are discussed and Sec. IV provides a comparison with theory. Section V concludes.

\section{EXPERIMENTAL SETUP}

The experimental setup has recently been described in detail $^{21}$ and only a brief description of the most important features is given here. A cold molecular beam $(\sim 1 \mathrm{~K})$ is formed by heating less than $50 \mathrm{mg}$ of solid 3,5-difluoro- $3^{\prime}, 5^{\prime}$ dibromo-4'-cyanobiphenyl in 90 bar of He to $170{ }^{\circ} \mathrm{C}$ and expanding the mixture into vacuum through a pulsed EvenLavie valve. This chemical is not commercially available and was synthesized specially for the experiment (see Appendix). Here, it should be noted that in the previously reported experiment, the molecular sample was slightly contaminated which can be seen directly from close inspection of the NMR spectrum in Fig. 4(d) of Ref. 15. In particular, the NMR spectrum shows a small impurity (5\%) of the biproduct, where both phenyl rings have flourine substituents. This contamination is problematic since the vapor pressure of the biproduct is higher than the target molecule and, therefore, the ratio between impurity and target molecules will be higher in the molecular beam than the initial $5 \%$ in the powder sample. Furthermore, the second most polarizable axis of the impurity will be located halfway between the two phenyl rings and, therefore, contribute to the significant blurring of the $\mathrm{F}^{+}$ion images and, thus, reduce the capability to experimentally resolve the torsional motion.

The beam of cold molecules passes through an electrostatic deflector that deflects the molecules according to their effective dipole moment. The effective dipole moment is determined by its rotational quantum state. ${ }^{20}$ Following deflection, the molecules enter a velocity map imaging (VMI) spectrometer (Fig. 1) where they are irradiated by three pulsed laser beams. The first pulse (YAG pulse: $\lambda=1064 \mathrm{~nm}$, $\left.\tau_{\mathrm{FWHM}}=10 \mathrm{~ns}, \mathrm{I}_{\mathrm{YAG}}=6 \times 10^{11} \mathrm{~W} / \mathrm{cm}^{2}\right)$ serves to adiabatically align the molecules. It is elliptically polarized with the major axis parallel to the spectrometer axis, the Z-axis (see Fig. 1). The second pulse (kick pulse: $800 \mathrm{~nm}, 200 \mathrm{fs}$, $\mathrm{I}_{\text {kick }}=2 \times 10^{13} \mathrm{~W} / \mathrm{cm}^{2}$ ) initiates torsion and it is linearly polarized along the Y-axis. The third pulse (probe pulse: $800 \mathrm{~nm}, 30 \mathrm{fs}, I_{\text {probe }}=3 \times 10^{14} \mathrm{~W} / \mathrm{cm}^{2}$ ) is used to characterize the spatial orientation of the two phenyl planes by Coulomb exploding the molecules and recording the recoiling $\mathrm{F}^{+}$and $\mathrm{Br}^{+}$fragments. It is linearly polarized either along the Z-axis or the Y-axis. By recording the series of images for different kick-probe delays, $t$, the evolution of the torsional motion can be monitored. To ensure that the molecules probed are both aligned and torsionally excited, the spot size of the three laser pulses are adjusted such that the 


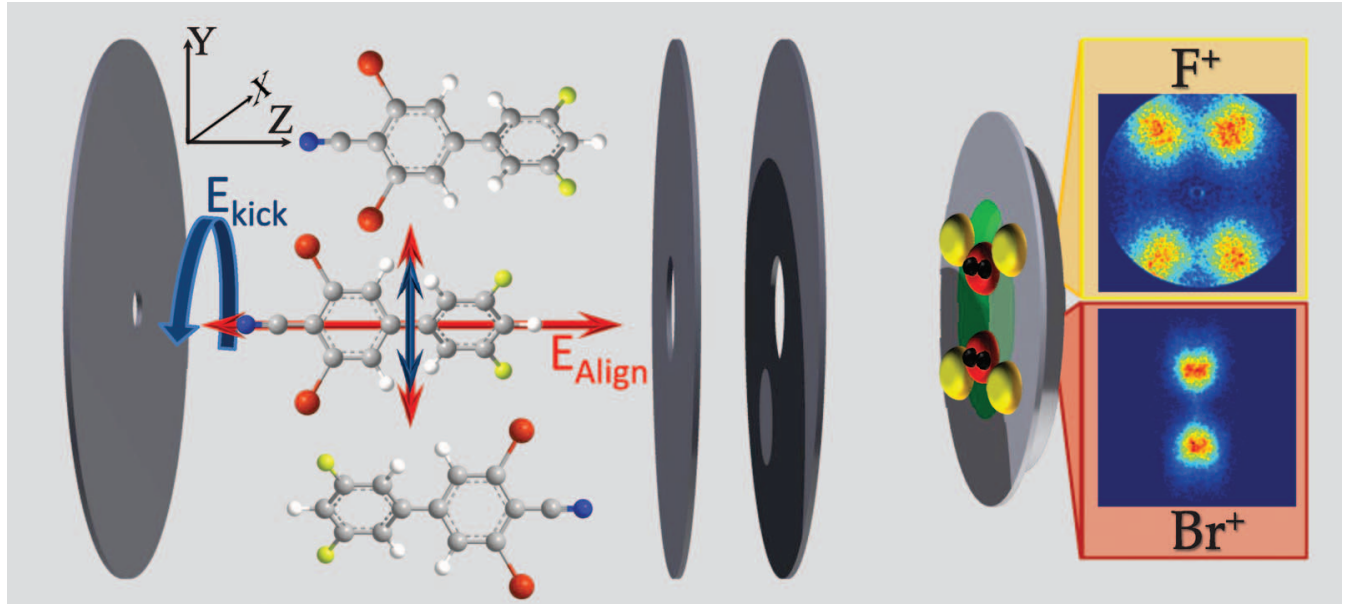

FIG. 1. Schematic of the velocity map imaging spectrometer used to detect $\mathrm{F}^{+}$and $\mathrm{Br}^{+}$ions. For illustrative purposes, the regions on the detector screen where $\mathrm{F}^{+}\left(\mathrm{Br}^{+}\right)$ions are expected to hit for perfectly aligned molecules, at equilibrium, are marked in yellow (black). The images behind the detector are experimental data recorded $2 \mathrm{ps}$ after the kick pulse. The polarization state of the YAG (alignment) pulse $\left(\lambda=1064 \mathrm{~nm}, \tau_{\mathrm{FWHM}}=10 \mathrm{~ns}, \mathrm{I}_{\mathrm{YAG}}=6 \times 10^{11} \mathrm{~W} / \mathrm{cm}^{2}\right)$ and the kick pulse $\left(800 \mathrm{~nm}, 200 \mathrm{fs}, \mathrm{I}_{\text {kick }}=2 \times 10^{13} \mathrm{~W} / \mathrm{cm}^{2}\right)$ are also displayed.

foci of the YAG and kick pulses are large $\left(\omega_{0}=38 \mu \mathrm{m}\right.$ and $\left.\omega_{0}=35 \mu \mathrm{m}\right)$ compared to the probe pulse $\left(\omega_{0}=26 \mu \mathrm{m}\right) .^{22}$

\section{EXPERIMENTAL RESULTS}

\section{A. Deflection}

The effect of the deflector on the molecular beam is shown in Fig. 2 by the vertical intensity profiles. They are obtained by recording the magnitude of an ion signal, produced by the probe pulse only, as a function of the vertical position of the laser focus similar to what was previously reported (see, for instance, Refs. 17, 19, and 21). Here, we use the strongest peak in the ion time-of-flight-spectrum, which is the $\mathrm{C}^{+}$signal. When the deflector is off, the molecular beam extends over $\sim 1.5 \mathrm{~mm}$, mainly determined by the diameter of the skimmer before the deflector. When the deflector is turned on, the molecular beam profile broadens and shifts upwards (positive Y-values). The molecules in the lowest rotational

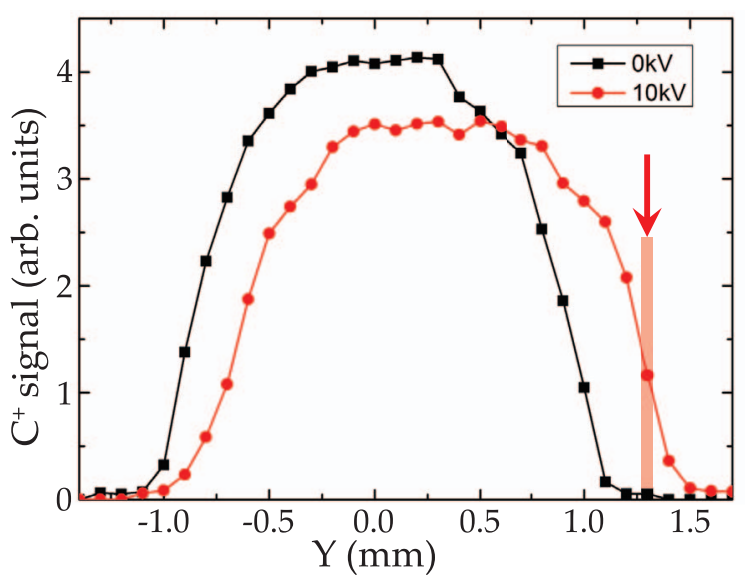

FIG. 2. Vertical profiles of the molecular beam measured by recording the $\mathrm{C}^{+}$signal as a function of the vertical position of the probe beam focus. The experimental data are shown by black squares (deflector off, $0 \mathrm{kV}$ ) and red circles (deflector on, $10 \mathrm{kV}$ ). The red arrow at $\mathrm{Y}=1.3 \mathrm{~mm}$ indicates the position of the laser foci used to acquire ion images of the deflected molecules. quantum states have the largest effective dipole moments and are, therefore, deflected the most, as shown in recent work on iodobenzene and benzonitrile molecules. ${ }^{19,20}$ In the measurements described below, experiments were conducted on quantum state-selected molecules simply by positioning the laser foci close to the upper cut-off region in the $10 \mathrm{kV}$ profile, indicated by the red arrow in Fig. $2(\mathrm{Y}=1.3 \mathrm{~mm})$. Our motivation for using the deflected part of the beam is that despite the density of molecules is lower, the alignment is higher. ${ }^{17,19,20}$

\section{B. Adiabatic prealignment}

The 3,5-difluoro-3',5'-dibromo-4'-cyanobiphenyl $\left(\mathrm{C}_{13} \mathrm{H}_{5} \mathrm{~F}_{2} \mathrm{Br}_{2} \mathrm{~N}\right.$, to be denoted by DFDBrCNBph in the following) is a chiral asymmetric top molecule, and the molecular composition in the supersonic expansion is a racemate, i.e., consisting of $50 \%$ of each of the two enantiomers.

Three-dimensional alignment of the molecules is achieved using an elliptically polarized YAG pulse which confines both the most polarizable axis (MPA) and the second most polarizable axis (SMPA) of the molecule, ${ }^{16,17,23,24}$ respectively. In DFDBrCNBph, the MPA is located along the stereogenic axis $(\mathrm{C}-\mathrm{C}$ bond axis connecting the two phenyl rings (see Fig. 1)), whereas the SMPA is perpendicular to this at an angle of $10^{\circ}$ from the phenyl-plane with the nitrile and bromine substituents towards the other phenyl ring (see Fig. 4). The effect of applying the YAG pulse, having its major and minor polarization axis along the Z-axis and the Y-axis, respectively, can be seen in panels (a) and (b) at $t=-0.33$ ps in Fig. 3, showing $\mathrm{F}^{+}$and $\mathrm{Br}^{+}$ion images from Coulomb exploding the molecules at the peak of the YAG pulse. In both images, two distinct features are observed. First, the center of the detector is almost void of ions (a minor region has been cut in the $\mathrm{F}^{+}$images to exclude a contamination from residual water molecules in the chamber). This observation is only compatible with the stereogenic axis (C-C bond axis) being confined perpendicular to the detector plane, i.e., along the Z-axis. Second, it is seen that both the 


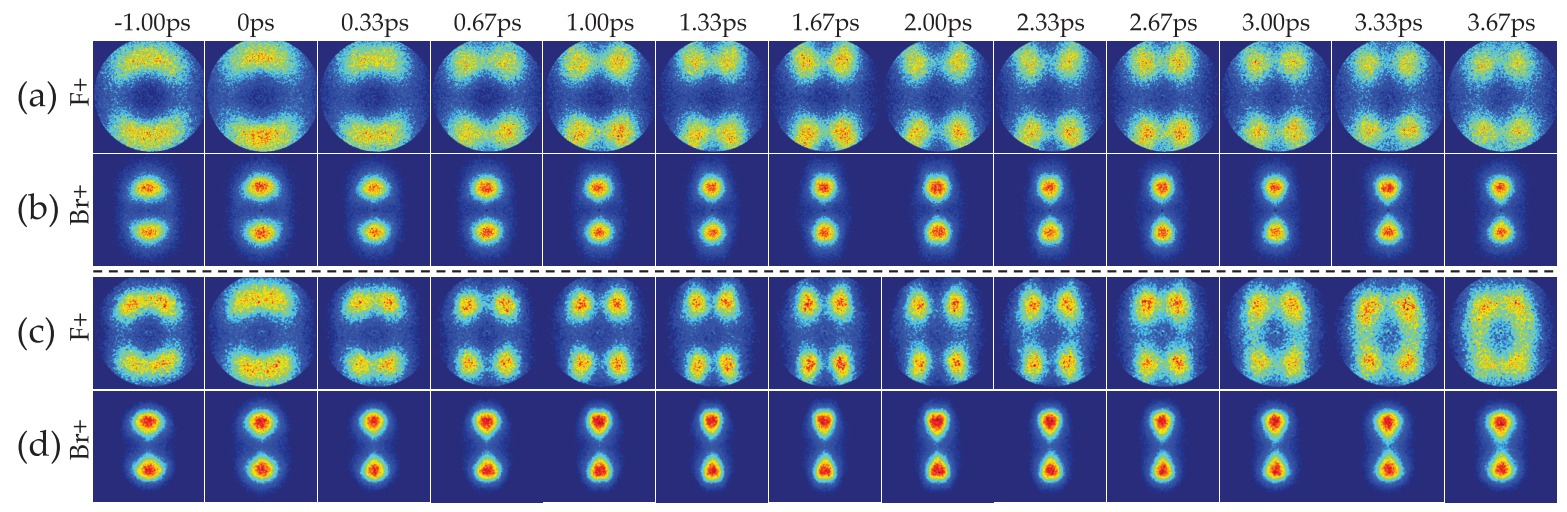

FIG. 3. $\mathrm{F}^{+}$(panels (a) and (c)) and $\mathrm{Br}^{+}$(panels (b) and (d)) ion images obtained as a function of the kick-probe time delay indicated in ps above each column of panels. Panels (a) and (b) and (c) and (d) were obtained using a linearly polarized probe pulse being perpendicular to (parallel with) the detector. The delay of the probe pulse $\left(\lambda=800 \mathrm{~nm}, \tau_{\mathrm{FWHM}}=30 \mathrm{fs}\right.$, $\left.\mathrm{I}_{\text {probe }}=3 \times 10^{14} \mathrm{~W} / \mathrm{cm}^{2}\right)$ with respect to the kick pulse $\left(\lambda=800 \mathrm{~nm}, \tau_{\mathrm{FWHM}}=200 \mathrm{fs}, \mathrm{I}_{\mathrm{kick}}=2 \times 10^{13}\right.$ $\mathrm{W} / \mathrm{cm}^{2}$ ) is given by the numbers on the top of the vertical panels.

$\mathrm{Br}^{+}$and the $\mathrm{F}^{+}$ions localize around the minor polarization axis of the YAG pulse (the Y-axis), the confinement being more pronounced for the $\mathrm{Br}^{+}$ions. We interpret this as the SMPA of the molecule being aligned along the Y-axis. The more spread-out signal of the $\mathrm{F}^{+}$ions results from the fact that the F-phenyl ring, at equilibrium, is offset $28^{\circ}$ from the SMPA, whereas the Br-phenyl ring is only offset by $10^{\circ}$. Thus, the joint observations of the $\mathrm{F}^{+}$and $\mathrm{Br}^{+}$images demonstrate that the molecules are $3 \mathrm{D}$ aligned as expected.

\section{Time-dependent torsion}

To investigate the torsional motion induced by the kick pulse, we recorded series of both $\mathrm{F}^{+}$and $\mathrm{Br}^{+}$images at different kick-probe delays. The probe pulse was polarized perpendicular to the detector plane to ensure a detection efficiency that is independent of the orientation of the F- and Br-phenylrings. The results are shown in panels (a) and (b) of Fig. 3. The first striking observation is that from $0.67 \mathrm{ps}$ to $4.0 \mathrm{ps}$, the $\mathrm{F}^{+}$images develop a pronounced 4-peak structure consisting of a pair in the top and a pair in the bottom part of the images. This shows that the kick pulse sharpens the alignment of the SMPA in agreement with a recent study on naphthalene, where it was demonstrated that the combined action of 3D adiabatic prealignment and a short kick pulse could significantly improve the alignment of the molecular plane in a time interval shortly after the kick pulse ${ }^{25}$ The second observation is that the angular separation of the two regions in each $\mathrm{F}^{+}$pair oscillates as a function of time. At $t=1.33 \mathrm{ps}$ the angular separation reaches a local minimum, at $t=2.0 \mathrm{ps}$ a local maximum, at $t=2.67 \mathrm{ps}$ a local minimum, etc. Turning to the $\mathrm{Br}^{+}$ion images, it is seen that the confinement along the Y-axis (vertical) improves. Unlike the $\mathrm{F}^{+}$images, a clear 4-peak structure is not observed. We ascribe this to the fact that the Br-phenyl ring is much closer to the SMPA, i.e., $\mathrm{Br}^{+}$ ions from molecules with the $\mathrm{Br}$-phenyl ring localized on each side of the SMPA will overlap and prevent a distinct 4-peak structure. It is, however, observed that the width of the $\mathrm{Br}^{+}$ oscillates as a function of time in phase with that observed in the $\mathrm{F}^{+}$images. These joint observations show that the dihedral angle between the Br-phenyl ring and the F-phenyl ring changes as a function of time. In other words, the torsional motion is directly imprinted on the ion distributions recorded.

The contrast can be improved significantly by recording the $\mathrm{F}^{+}$and $\mathrm{Br}^{+}$images with the probe pulse polarized parallel to the kick pulse polarization. These data are displayed in panels (c) and (d) in Fig. 3. The evolution of both ion species is the same as that observed in rows (a) and (b) in Fig. 3, but the contrast is significantly higher. In particular, the 4-peak structure in the $\mathrm{F}^{+}$images is very distinct with complete separation between the two regions in each pair and the oscillations in the angular width of the $\mathrm{Br}^{+}$images stand out very clearly. Our qualitative understanding of the improved contrast is that the probe pulse preferentially ionizes molecules with their SMPA close to its polarization axis. In other words, the probe pulse selects the molecules with the strongest alignment of the SMPA.

The quantitative analysis is performed by fitting the angular distributions, extracted from the ion images, with a sum of four Gaussian functions. From these fits, the average angles, $\left\langle\phi_{\mathrm{Br}^{+}}\right\rangle$, of the Br-phenyl ring, with respect to the kick pulse polarization, can be extracted directly from the peak positions of the Gaussian functions. Similarly, the average angle, $\left\langle\phi_{\mathrm{F}^{+}}\right\rangle$, of the F-phenyl-ring, can be found from the fit of the $\mathrm{F}^{+}$angular distributions.

Figure 4 displays the average angle between the Fphenyl-rings (black squares) and the kick pulse polarization as a function of kick-probe time delay for the time interval, where a clear four-peak-structure can be identified in the angular distributions. The dashed black line splined to the experimental measurements indicates that the oscillations observed in the images of the F-phenyl ring have an amplitude of $\sim 2.5^{\circ}$ with respect to the kick pulse polarization direction and a period of $1.25 \mathrm{ps}$. Closer examination of the oscillating trace reveals a small overall increase towards larger $\left\langle\phi_{\mathrm{F}^{+}}\right\rangle$, which shows that the plane of the F-phenyl-ring slowly moves away from the kick pulse polarization. Also, the average angle between the Br-phenyl-rings (red circles) and the kick pulse polarization is shown. In the time interval $1-4 \mathrm{ps}$, the uncertainties on the measurements are small and allow for the determination of the period $(1.25 \mathrm{ps})$ and amplitude $\left(\sim 0.5^{\circ}\right)$ of the motion. For the Br-phenyl-ring, we observe a small 
(a)

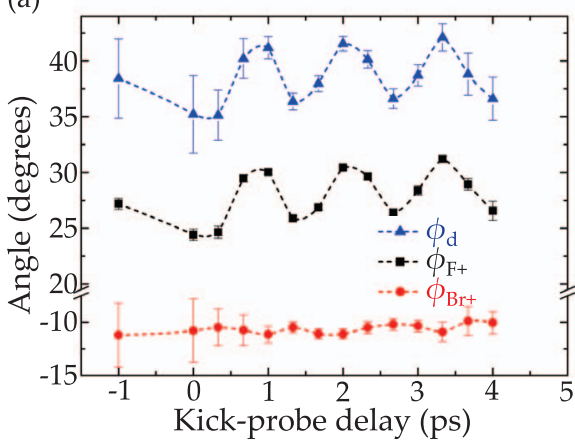

(b)

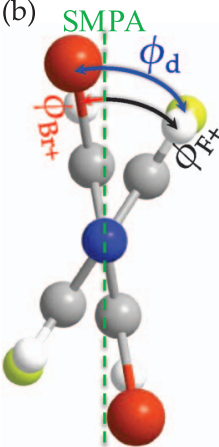

FIG. 4. (a) Time-dependence of the dihedral angle (blue triangles) following the kick pulse, obtained by adding the orientation of the F-phenyl-ring (black squares) and the orientation of the Br-phenyl-ring with respect to the SMPA - see text for details. (b) Sketch of the molecular structure as seen in end view. The SMPA of the molecule and the extracted angles are marked on the drawing.

decrease in the $\left\langle\phi_{\mathrm{Br}^{+}}\right\rangle$with increasing time delay. The slight decrease in $\left\langle\phi_{\mathrm{Br}^{+}}\right\rangle$and corresponding increase in $\left\langle\phi_{\mathrm{F}^{+}}\right\rangle$are fully compatible with an overall rotation of the molecule about the stereogenic axis.

The average values, $\left\langle\phi_{\mathrm{F}^{+}}\right\rangle$and $\left\langle\phi_{\mathrm{Br}^{+}}\right\rangle$, can be used to calculate the instantaneous dihedral angle, $\phi_{\mathrm{d}}$, of the molecule by simple addition (see Fig. 4(b)). By retrieving these average values at every kick-probe time delay, it is possible to directly monitor the evolution of the dihedral angle of the molecule. The results for the dihedral angle ( $\phi_{\mathrm{d}}$, blue triangles) are displayed in Fig. 4(a), with a dashed line included to help guide the eye and emphasize the torsional motion. The oscillation is seen to have an amplitude of $3^{\circ}$ and a period of $1.25 \mathrm{ps}$.

We also recorded $\mathrm{F}^{+}$and $\mathrm{Br}^{+}$ion images for times larger than 4 ps. The results, including the 3.0 ps data, are shown in Fig. 5. For $t>4.0 \mathrm{ps}$, the $\mathrm{F}^{+}$ion images loose their distinct four region structure and, instead, evolve into a circular shape at $t \geq 8 \mathrm{ps}$. This prevents tracking the evolution of $\left\langle\phi_{\mathrm{F}^{+}}\right\rangle$. The images, however, confirm that the stereogenic axis of the molecule is still tightly confined along the major axis of the YAG polarization ellipse, since the central region is absent from ions. The corresponding $\mathrm{Br}^{+}$ion images show a similar delocalization behavior, but the broadening is slower. This observation is consistent with theory as discussed in Sec. IV B.

To quantify the time-dependence of the delocalization in the $\mathrm{F}^{+}$and $\mathrm{Br}^{+}$ion images we determined $\left\langle\cos ^{2} \alpha_{2 D}\right\rangle$ for each image, where $\alpha_{2 D}$ is the angle between the projection of an

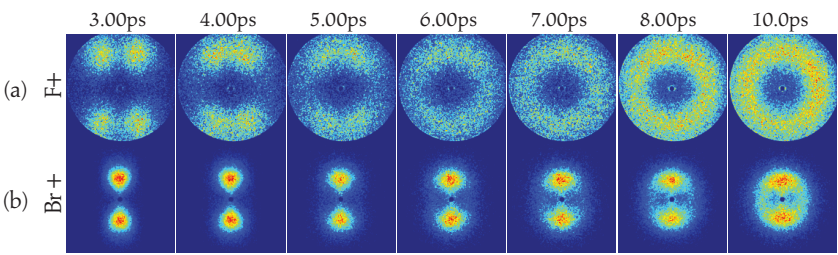

FIG. 5. $\mathrm{F}^{+}$(a) and $\mathrm{Br}^{+}$(b) ion images obtained at long $(t \geq 3 \mathrm{ps})$ kick-probe time delays obtained using a probe pulse linearly polarized perpendicular to the detector. The delay of the probe pulse $\left(\lambda=800 \mathrm{~nm}, \tau_{\mathrm{FWHM}}=30 \mathrm{fs}\right.$, $\left.\mathrm{I}_{\text {probe }}=3 \times 10^{14} \mathrm{~W} / \mathrm{cm}^{2}\right)$ with respect to the kick pulse $\left(\lambda=800 \mathrm{~nm}, \tau_{\mathrm{FWHM}}\right.$ $=200 \mathrm{fs}, \mathrm{I}_{\mathrm{kick}}=2 \times 10^{13} \mathrm{~W} / \mathrm{cm}^{2}$ ) is given by the numbers on the top of the vertical panels.

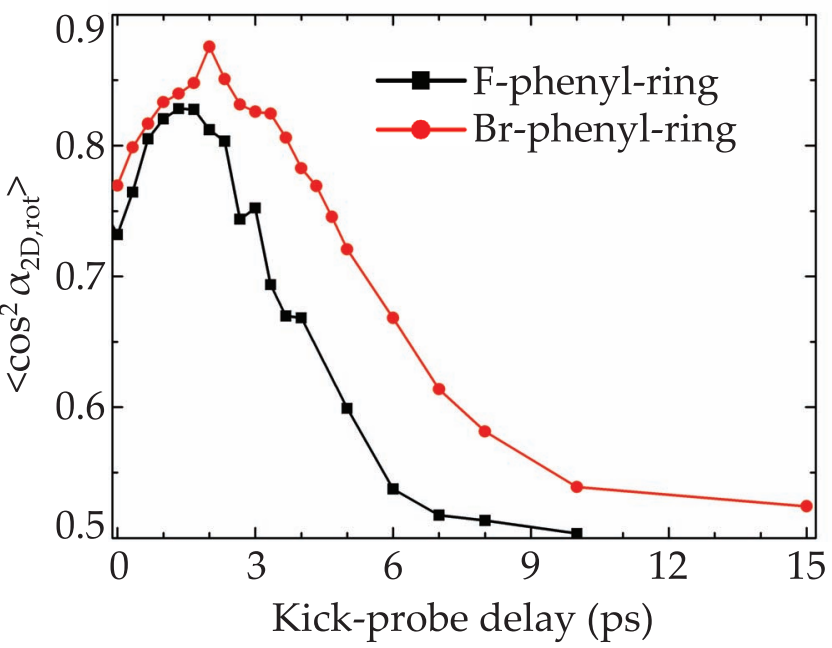

FIG. 6. Time dependence of the planar alignment of the individual phenylrings. The $\left\langle\cos ^{2} \alpha_{2 D}\right\rangle$ values are calculated from rotated angular distributions to remove the effect of the torsion (see text for details). Black squares (red circles) correspond to the confinement of the F-phenyl-ring (Br-phenylring).

ion velocity vector on the detector plane and the Y-axis after rotating the ion distributions such that the center of the fitted Gaussians coincides with the Y-axis. This is implemented by rotating the peaks fitted to the angular distributions of $\mathrm{F}^{+}$and $\mathrm{Br}^{+}$ions by the following procedure: If the peak is located in the first or third quadrant, the $\mathrm{F}^{+}\left(\mathrm{Br}^{+}\right)$image is rotated by $-\left\langle\phi_{\mathrm{F}^{+}}\right\rangle\left(-\left\langle\phi_{\mathrm{Br}^{+}}\right\rangle\right)$before the calculation of $\left\langle\cos ^{2} \alpha_{2 D}\right\rangle$, whereas if the peak is in the second or fourth quadrant, the image is rotated by $+\left\langle\phi_{\mathrm{F}^{+}}\right\rangle\left(+\left\langle\phi_{\mathrm{Br}^{+}}\right\rangle\right)$. This procedure allows for the calculation of the best estimate of the spread from the mean by including all data. The $\left\langle\cos ^{2} \alpha_{2 D}\right\rangle$ values are displayed in Fig. 6. A clear difference in the delocalization dynamics of the two phenyl planes is observed, with the light fluorine substituted phenyl ring approaching $\left\langle\cos ^{2} \alpha_{2 D}\right\rangle$ $=0.5$ more rapidly than the heavier bromine substituted phenyl ring. This is fully consistent with semi-classical theoretical predictions presented in Sec. IV B.

\section{Covariance and autovariance mapping}

Before discussing the theoretical results, we introduce covariance mapping analysis ${ }^{26}$ to the experimental data. This analysis strengthens our interpretation of torsional motion, based on the fluorine and bromine ion images in Fig. 3. Covariance mapping is a technique to reveal correlations, which in the high count rate regime would otherwise become blurred or completely lost due to the large contributions from uncorrelated events. The basic principle is to calculate the covariance, i.e., to obtain a cross-correlation of the variance in the data, ${ }^{27}$ to extract the deviation from the mean value for all the individual frames of the ion image, pertaining to each (probe) laser shot. This is fundamentally different from coincidence measurements in, e.g., COLTRIMS and reactionmicroscopes ${ }^{28,29}$ where the count rate is restricted to less than one event per laser shot, since the covariance map is produced from the correlations of the variance, which per definition 
has no restrictions on the number of events obtained per laser shot.

The covariance $(C(x, y))$ between two observables $X(x)$ and $Y(y)$ is defined as the mean of the product between the observables $\langle X(x) Y(y)\rangle$ subtracted by the product of the means $\langle X(x)\rangle\langle Y(y)\rangle$ :

$$
\begin{aligned}
C(x, y) & =\langle(X(x)-\langle X(x)\rangle)(Y(y)-\langle Y(y)\rangle)\rangle \\
& =\langle X(x) Y(y)\rangle-\langle X(x)\rangle\langle Y(y)\rangle .
\end{aligned}
$$

Here, $X$ and $Y$ correspond to the observed signals, with $x$ and $y$ being the variables investigated for correlation.

Although many different correlation techniques have been explored previously and found many different applications within natural science, ${ }^{29-32}$ to our knowledge, no reports on using covariance mapping to extract angular correlation information from 2D ion images has been reported. We suggest to do this by replacing $X$ and $Y$ with $\Theta$ and $\Xi$, corresponding to the angular distributions of the ion signal, and $x$ and $y$ with $\theta$ and $\xi$ being the ion detection angle with respect to the Y-axis (Fig. 1). Applying this version of covariance mapping to the impact coordinates of the ions in the $2 \mathrm{D}$ velocity map images allows for the extraction of the angular correlation between ejected fragments. In our experiment, only one ion species, e.g., $\mathrm{F}^{+}$, is detected per laser shot and thus $\Theta$ $=\Xi$. As a consequence, Eq. (1) may be written as

$$
\begin{aligned}
C\left(\theta_{1}, \theta_{2}\right)= & \left\langle\Theta\left(\theta_{1}\right) \Theta\left(\theta_{2}\right)\right\rangle-\left\langle\Theta\left(\theta_{1}\right)\right\rangle\left\langle\Theta\left(\theta_{2}\right)\right\rangle \\
= & \frac{1}{N} \sum_{i=1}^{N} \Theta_{i}\left(\theta_{1}\right) \Theta_{i}\left(\theta_{2}\right)-\left[\frac{1}{N} \sum_{i=1}^{N} \Theta_{i}\left(\theta_{1}\right)\right] \\
& \times\left[\frac{1}{N} \sum_{i=j}^{N} \Theta_{j}\left(\theta_{2}\right)\right] .
\end{aligned}
$$

Since this corresponds to investigating correlations of a signal with itself, this approach is equivalent to obtaining an autocorrelation of the variance in the data and so the 2D maps depicting the correlation should be termed autovariance maps. The principle of autovariance mapping is illustrated in Fig. 7. Following each laser shot the ions focused onto the detector are

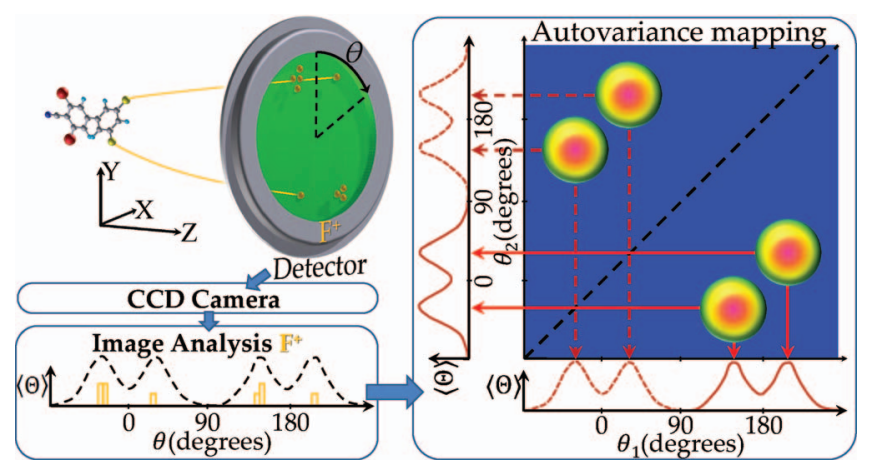

FIG. 7. The autovariance mapping principle illustrated for $\mathrm{F}^{+}$fragments produced by coulomb explosion of laser aligned DFDBrCNBph. From the autovariance map seen on the right, it is possible to extract correlation information between the ejection angle of the $\mathrm{F}^{+}$ions. The blue arrows indicate the steps in the data acquisition and data processing leading to the production of autovariance maps. recorded by a CCD camera after which an image analysis program saves the impact coordinates, illustrated by the yellow bars in the image analysis box. The experiment is repeated $N$ times, typically $N=10.000$, giving the averaged ion images (such as those shown in Fig. 3) with the averaged angular distributions (Fig. 7 dashed black line). The autovariance $C\left(\theta_{1}\right.$, $\theta_{2}$ ) can now be determined by applying Eq. (2) to all $N$ frames of the ion image, and displayed as a 2D map of the ejection angles. In this map, the two axes correspond to the angular distribution from the averaged image, with the positive Y-axis denoting $0^{\circ}$.

Autovariance maps obtained through this procedure will tend to produce a strong line along the diagonal of the map, since the observation of an ion in frame $\Theta_{i}$ at the angle $\theta_{1}$ is naturally also observed in the same frame, for the identical observable $\Theta_{i}$, at $\theta_{2}=\theta_{1}$. The diagonal, which is equivalent to the square of the rms standard deviation by definition, will also act as an axis of symmetry for the correlation, since $C\left(\theta_{1}, \theta_{2}\right)=C\left(\theta_{2}, \theta_{1}\right)$. The positive areas of the autovariance maps, which fall outside the diagonal $\left(\theta_{1} \neq \theta_{2}\right)$, indicate the correlation between ions at $\theta_{1}$ and $\theta_{2}$, showing an increased likelihood of observing an ion at $\theta_{2}$ provided that an ion is detected at $\theta_{1}$.

Figure 8 shows the angular autovariance maps obtained from both $\mathrm{F}^{+}$and $\mathrm{Br}^{+}$ion images at two different kick-probe time delays. In these maps, the dominant autocorrelation signal along the diagonal has been set to zero to increase the contrast of the interesting but less intense correlations in the autovariance map. Also these data sets have been binned in steps of $5^{\circ}$ to smoothen out small fluctuations. A striking feature of all autovariance maps is that the observed correlations are restricted to areas where the angle between the ejected

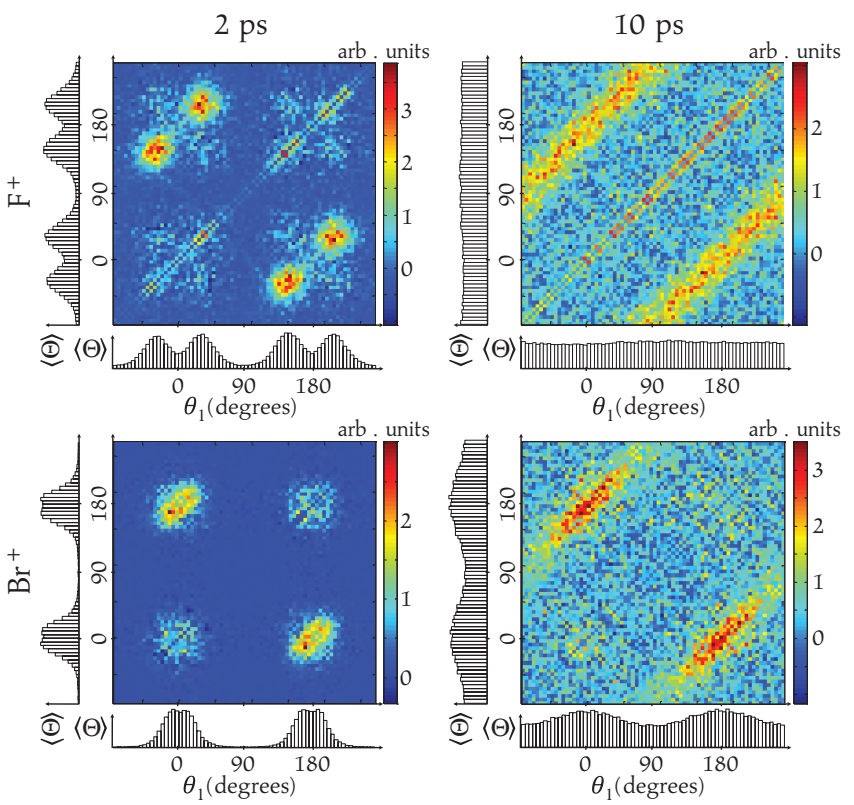

FIG. 8. Autovariance maps for $\mathrm{F}^{+}$(upper panels) and $\mathrm{Br}^{+}$ions (lower panels) at kick-probe time delays of 2 ps (left column) and 10 ps (right column). To smoothen out small fluctuations, the data have been binned in steps of $5^{\circ}$. Also the large signal, due to autocorrelation, along the diagonal of the maps, has been set to zero to reveal the less intense correlations in the autovariance map. 
ions is close to $180^{\circ}$. This is seen, e.g., in the $\mathrm{F}^{+}$map at $2 \mathrm{ps,}$ where the signal at $\sim 150^{\circ}\left(\sim 210^{\circ}\right)$ is correlated to the region at $\sim-30^{\circ}\left(\sim 30^{\circ}\right)$. In other words, when an $\mathrm{F}^{+}$ion is ejected upwards at an angle of $150^{\circ}$, there is a high probability that another $\mathrm{F}^{+}$ion is ejected at $-30^{\circ}$, i.e., just in the opposite direction. This corroborates our interpretation of the two ion species as direct observables of the orientation of each of the phenyl rings. In addition, the observation of four prominent positive areas in the autovariance map at $t=2 \mathrm{ps}$ confirms the interpretation of the 4-peak structure in the $\mathrm{F}^{+}$ion images (Fig. 3). Similar considerations hold for the $\mathrm{Br}^{+}$autovariance map at $t=2 \mathrm{ps}$. Notably, the appearance of a 4-peak structure with positive covariance shows even clearer than the averaged ion image on Fig. 3, that the Br-phenyl plane has a small angular offset with respect to the kick pulse polarization. At $t=10 \mathrm{ps}$, there is no longer a 4-peak structure but the distinct oblique lines show unambiguously that emission of both $\mathrm{F}^{+}$and $\mathrm{Br}^{+}$ions still occurs in a pairs with an upward and a downward ion. The extension of the covariance principle to obtain angular correlation between ions allows for the extraction of additional information from the $2 \mathrm{D}$ images obtained using VMI spectrometers. As shown here, a single detector setup, can be used to obtain autovariance maps between ions of identical mass-to-charge ratios, which can be used to both substantiate and clarify the interpretation of the recorded ion images. This can be extended to obtain the correlation between ions with different mass-to-charge ratios by pulsing the front of the MCP twice per laser shot and running the CCD camera in burst mode. In addition, we note that the technique can also be extended to obtain radial autovariance maps, for fragmentation processes with multiple energy channels, or combined to encompass both angular and radial correlation of the $2 \mathrm{D}$ ion images.

\section{COMPARISON WITH THEORY}

The main findings of Sec. III were that the dihedral angle oscillates with a period of approximately $1.25 \mathrm{ps}$, and an amplitude of $3^{\circ}$. These findings were based on an analysis of the data assuming that the two ion species move out to the detector in the plane of the (substituted) phenyl ring from which they originated. The covariance and autovariance analysis introduced validated this assumption and we may build the theory on this fact.

\section{A. Early time dynamics: Torsional motion}

The purpose of this section is to provide a theoretical foundation for understanding the experimentally observed ion images shown in Fig. 3. Atomic units $\left(|e|=\hbar=m_{\mathrm{e}}=a_{0}\right.$ $=1$ ) are used throughout. A useful starting point is to discuss the widths of the ion images. These may conveniently be quantified in terms of the variance

$$
\sigma_{\mathrm{i}}^{2}=\left\langle\phi_{\mathrm{i}}^{2}\right\rangle-\left\langle\phi_{\mathrm{i}}\right\rangle^{2}, \quad \mathrm{i}=\mathrm{Br}^{+}, \mathrm{F}^{+}
$$

For the sake of interpretation, we rewrite these two angles in terms of the dihedral angle, $\phi_{\mathrm{d}}$, and the overall rotation, $\Phi$

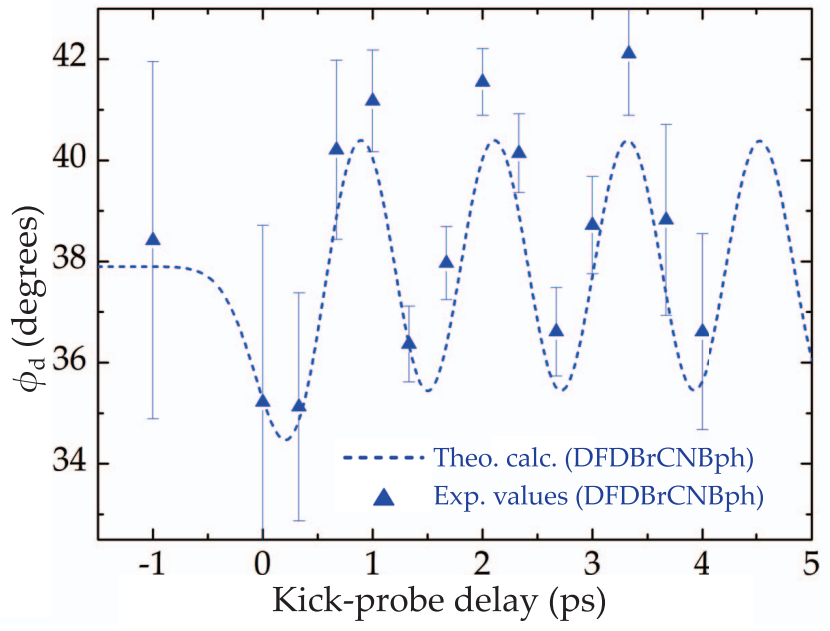

FIG. 9. Comparison: Blue triangles-experimental results, dashed blue line-theoretical calculation on DFDBrBPh shifted by $-0.9^{\circ}$ to match the dihedral angle of DFDBrCNBPh at equilibrium.

[see Fig. 4(b)]:

$$
\begin{gathered}
\phi_{\mathrm{Br}^{+}}=\Phi+\eta \phi_{\mathrm{d}}, \\
\phi_{\mathrm{F}^{+}}=\Phi-(1-\eta) \phi_{\mathrm{d}}, \\
\eta=\frac{I_{\mathrm{F}^{+}}}{I_{\mathrm{F}^{+}}+I_{\mathrm{Br}^{+}}} .
\end{gathered}
$$

Consequently, we may express the variance of the observed ions as

$$
\begin{aligned}
\sigma_{\phi_{\mathrm{Br}^{+}}}^{2} & =\sigma_{\Phi}^{2}+\eta^{2} \sigma_{\phi_{\mathrm{d}}}^{2} \\
& +2 \eta\left(\left\langle\Phi \phi_{\mathrm{d}}\right\rangle-\langle\Phi\rangle\left\langle\phi_{\mathrm{d}}\right\rangle\right), \\
\sigma_{\phi_{\mathrm{F}^{+}}}^{2}= & \sigma_{\Phi}^{2}+(1-\eta)^{2} \sigma_{\phi_{\mathrm{d}}}^{2} \\
& -2(1-\eta)\left(\left\langle\Phi \phi_{\mathrm{d}}\right\rangle-\langle\Phi\rangle\left\langle\phi_{\mathrm{d}}\right\rangle\right) .
\end{aligned}
$$

Previous investigations show that the variance of the dihedral angle is small (see Figs. 3 and 9 in Ref. 15). It is therefore obvious to ask if $\sigma_{\Phi}^{2} \gg \sigma_{\phi_{\mathrm{d}}}^{2}$. As follows from Eqs. (7) and (8), this can only be the case if the widths of the ion images are comparable. Inspection of Figs. 5 and 6 confirms that for long times $(t>3 \mathrm{ps})$, this is not true. For shorter times, however, the widths of the two ion images are close (see Fig. 3). Further, we have previously argued for separability of the $\Phi$ and $\phi_{\mathrm{d}}$ coordinates in this limit ${ }^{15}$ meaning that the last term in Eqs. (7) and (8) vanishes completely. In this regime, $\Phi$ is an adiabatic parameter and we simply discuss the short-term dynamics based on calculations with $\Phi$ fixed meaning that only torsion takes place.

The torsional motion is then dictated by the timedependent Schrödinger equation

$$
\begin{aligned}
& i \partial_{t} \Psi\left(\Phi ; \phi_{\mathrm{d}}, t\right) \\
& \quad=\left[T_{\mathrm{d}}+V_{\mathrm{tor}}\left(\phi_{\mathrm{d}}\right)+V_{\mathrm{kick}}\left(\Phi ; \phi_{\mathrm{d}}, t\right)\right] \Psi\left(\Phi ; \phi_{\mathrm{d}}, t\right),
\end{aligned}
$$


with

$$
T_{\mathrm{d}}=-\frac{I_{\mathrm{Br}^{+}}+I_{\mathrm{F}^{+}}}{2 I_{\mathrm{Br}^{+}} I_{\mathrm{F}^{+}}} \frac{\partial^{2}}{\partial \phi_{\mathrm{d}}^{2}}
$$

being the rotational kinetic energy due to torsion, $V_{\text {tor }}$ the torsional potential and $V_{\text {kick }}$ the polarizability interaction energy between the kick pulse and the molecule:

$$
\begin{aligned}
V_{\text {kick }}\left(\Phi, \phi_{\mathrm{d}}, t\right)= & -\frac{1}{4} F_{0}^{2}(t)\left[\alpha_{\mathrm{xx}}\left(\phi_{d}\right) \cos ^{2}\left(\Phi+\eta \phi_{\mathrm{d}}\right)\right. \\
& +\alpha_{\mathrm{yy}}\left(\phi_{\mathrm{d}}\right) \sin ^{2}\left(\Phi+\eta \phi_{\mathrm{d}}\right) \\
& \left.-2 \alpha_{\mathrm{xy}}\left(\phi_{\mathrm{d}}\right) \cos \left(\Phi+\eta \phi_{\mathrm{d}}\right) \sin \left(\Phi+\eta \phi_{\mathrm{d}}\right)\right] .
\end{aligned}
$$

We solve this equation for DFDBrBPh using a close coupling method with a value of $\Phi$ corresponding to the SMPA and kick pulse polarization aligned. As initial state $\Psi\left(t_{0}\right)$, we use a state localized in the torsional well at $39^{\circ}$. The details have been given in Ref. 15.

Figure 9 shows the result of the calculation. The torsional dynamics consists of small, periodic oscillations. As argued previously ${ }_{15}^{15}$ these oscillations stem from the fact that the kick pulse leaves DFDBrBPh in a coherent superposition of the ground state and first excited state of the torsional potentialessentially simple harmonic oscillator states separated by $3.42 \mathrm{meV}$. The molecule used in the experiment is DFDBrC$\mathrm{NBPh}$ rather than DFDBrBPh. The torsional potential differs, however, only slightly from that used previously for DFD$\mathrm{BrBPh}$. Since the interaction with the kick pulse only populates the ground and the first excited torsional states ${ }^{15}$ where the potential is very close to harmonic, the main effect to take into account is the shift of the minima of the torsional potential from one molecule to the other $\left(-0.9^{\circ}\right)$. The torsional dynamics, induced by the kick pulse, is determined by the two polarizability components $\left(\alpha_{\mathrm{xx}}\right.$ and $\left.\alpha_{\mathrm{yy}}\right)$ perpendicular to the most polarizable axis. Since these polarizability components are essentially identical for DFDBrCNBPh ${ }^{23}$ and DFDBrBPh, ${ }^{15}$ we may simply shift the previously calculated theoretical curve by $-0.9^{\circ}$ to obtain a comparison with the experimental results. As seen in Fig. 9, this leads to an impressive agreement with the experimental results.

\section{B. Long time behavior: Dephasing of the phenyl rings}

For longer time scales ( $t>3 \mathrm{ps})$, the semi-classical model is no longer attractive. The reasons are that the widths of the two ion images (Figs. 3 and 5) start to deviate from one another meaning that the variance is no longer dominated by $\Phi$ alone and, hence, that the separability in $\Phi$ and $\phi_{\mathrm{d}}$ breaks down. $^{12,15}$

To provide a qualitative analysis in this long-time regime, we exploit a different theoretical limit, where the phenylrings are treated as rigid rotors. In this case, each of the rings $\mathrm{i}=\mathrm{Br}^{+}, \mathrm{F}^{+}$are assigned their individual moments of inertia $\left(I_{\mathrm{i}}\right)$ and they can each be described by a wave packet

$$
\Psi_{\mathrm{i}}\left(\phi_{\mathrm{i}}, t\right)=\sum_{J_{\mathrm{i}}} c_{J_{\mathrm{i}}} \frac{1}{\sqrt{2 \pi}} e^{i J_{\mathrm{i}} \phi_{\mathrm{i}}} e^{-i \frac{J_{\mathrm{i}}^{2} t}{2 l_{\mathrm{i}}} t},
$$

where $J_{\mathrm{i}}$ labels a rotational state and $c_{J_{\mathrm{i}}}$ is a fixed constant after the end of kick pulse.

To estimate on what time scale, $\tau_{\mathrm{i}}$, such a wave packet dephases, we look at the time evolution of the coherence between two neighboring levels $J_{\mathrm{i}}$ and $J_{\mathrm{i}}+1$. It immediately follows from (12) that this coherence beats like

$$
\cos \left(\frac{2 J_{\mathrm{i}}+1}{2 I_{\mathrm{i}}} t\right) .
$$

Consequently, the two rings dephase at a ratio

$$
r=\frac{\tau_{\mathrm{F}^{+}}}{\tau_{\mathrm{Br}^{+}}}=\frac{I_{\mathrm{F}^{+}}}{I_{\mathrm{Br}^{+}}} \cdot \frac{2 J_{\mathrm{Br}^{+}}+1}{2 J_{\mathrm{F}^{+}}+1} .
$$

The value of $J_{i}$ depends on the rotational kinetic energy, $E_{\text {kin,i, }}$, of each ring. Due to the $a$ times higher polarizability of the Br-phenyl-ring compared to the F-phenyl-ring, we assume that $E_{\mathrm{kin}, \mathrm{Br}}=a \times E_{\mathrm{kin}, \mathrm{F}}$ with $E_{\mathrm{kin}, \mathrm{i}}=J_{\mathrm{i}}^{2} / 2 I_{\mathrm{i}}$. This result is achieved by integrating the torque of a classic rigid rotor from $0^{\circ}$ to $90^{\circ}$ to obtain an energy estimate. We then immediately have

$$
\frac{J_{\mathrm{Br}^{+}}}{J_{\mathrm{F}^{+}}}=\sqrt{a \frac{I_{\mathrm{Br}^{+}}}{I_{\mathrm{F}^{+}}} .}
$$

Using this in Eq. (14) yields

$$
r \simeq \sqrt{a \frac{I_{\mathrm{F}^{+}}}{I_{\mathrm{Br}^{+}}}} .
$$

With a value of 0.21 for the ratio of the moments of inertia and $a=2$ (see Ref. 18), we obtain $r=0.65$ in qualitative agreement with the dephasing ratio of 0.68 obtained from the data presented in Fig. 6 by comparing the full width at half maximum values of the Gaussian fits to the ion pictures.

\section{CONCLUSIONS}

In conclusion, we have shown that it is possible to induce torsion in a substituted biphenyl molecule with a nonresonant kick pulse and image the motion in real-time with an intense delayed probe pulse. The measurements show a distinct torsional motion with an amplitude of $3^{\circ}$ and a period of $1.25 \mathrm{ps}$ for the first $4 \mathrm{ps}$ after the kick pulse, in excellent agreement with results from our theoretical model. At longer times delocalization of the two phenyl rings of the molecule blurs torsion.

An important prerequisite for the experiment is the ability to keep the molecules fixed-in-space during the timeresolved experiment, practically obtained by $3 \mathrm{D}$, aligning them in the adiabatic limit. This places both benzene rings of the molecule perpendicular to a $2 \mathrm{D}$ imaging detector. Upon Coulomb explosion by the probe laser, $\mathrm{Br}^{+}$ions from one benzene ring imprint an ion image on the detector screen that uniquely identifies the orientation of this ring. Similarly, $\mathrm{F}^{+}$ion images identify the orientation of the second ring. The difference between the orientation of the two rings determines the dihedral angle, i.e., the normal vibrational coordinate characterizing torsion. Such a procedure would not be possible for samples of randomly oriented molecules. 
The paper also showed that it is possible to apply covariance mapping analysis of ion images. This revealed clear correlations between the emission direction of ions strengthening our interpretation of Coulomb explosion as a direct and unique observable of molecular orientation. Covariance and autovariance methods applied to ion imaging have a potential to provide additional information beyond what is normally extracted from averaged quantities.

\section{ACKNOWLEDGMENTS}

The work was supported by the Carlsberg Foundation and the Danish Council for Independent Research (Natural Sciences). C.B.M. acknowledges support from the Chemical Sciences, Geosciences, and Biosciences Division, Office of Basic Energy Sciences, Office of Science, US Department of Energy. M.P.J. was supported by a MICINN Juan de la Cierva research (Grant No. JCI-2009-05953), and the Academy of Finland (136079). L.B.M. was supported by the Lundbeck Foundation, the Danish Research Council (Grant No. 10085430) and ERC-StG (Project No. 277767-TDMET).

\section{APPENDIX: SYNTHESIS OF 3,5-difluoro-3',5'-dibromo- 4'-cyanobiphenyl}

3,5-Difluoro-4'-aminobiphenyl: ${ }^{33}$ To a sample, vial was added 4-bromoaniline $(172.0 \mathrm{mg}, 1.0 \mathrm{mmol})$, 3,5difluorophenyl boronic acid $(189.5 \mathrm{mg}, 1.2 \mathrm{mmol})$, tetrabutylammonium bromide $(32.4 \mathrm{mg}, 0.1 \mathrm{mmol})$, potassium carbonate $(276.4 \mathrm{mg}, 2 \mathrm{mmol})$, and water $(2 \mathrm{ml})$. To this was added $100 \mu \mathrm{l}$ of a $\mathrm{PdCl}_{2}:$ EDTA: $\mathrm{Na}_{2} \mathrm{CO}_{3}$ stock solution prepared by mixing $\mathrm{PdCl}_{2}(17.7 \mathrm{mg}, 0.1 \mathrm{mmol})$, EDTA $(37.2 \mathrm{mg}$, $0.1 \mathrm{mmol})$, and $\mathrm{Na}_{2} \mathrm{CO}_{3}(21.2 \mathrm{mg}, 0.2 \mathrm{mmol})$ in water $(1 \mathrm{ml})$. The vial which was fitted with a Teflon sealed screwcap and heated to $100{ }^{\circ} \mathrm{C}$ for $45 \mathrm{~min}$ after which, the reaction was cooled to room temperature. The mixture was diluted with $\mathrm{Et}_{2} \mathrm{O}(25 \mathrm{ml})$ and $\mathrm{CH}_{2} \mathrm{Cl}_{2}(10 \mathrm{ml})$. The organic phase was washed with water $(2 \times 15 \mathrm{ml})$, brine $(15 \mathrm{ml})$, and dried using $\mathrm{MgSO}_{4}$. The solvents were removed under reduced pressure and the crude reaction mixture was purified by column chromatography eluting with $\mathrm{CH}_{2} \mathrm{Cl}_{2}$ to afford the title compound as a colorless solid $(170.5 \mathrm{mg}, 83 \%) .{ }^{1} \mathrm{H}$ NMR $(400 \mathrm{MHz}$, $\left.\mathrm{CDCl}_{3}\right) \delta(\mathrm{ppm}) 7.37(\mathrm{~d}, 2 \mathrm{H}, \mathrm{J}=8.2 \mathrm{~Hz}), 7.07-7.00(\mathrm{~m}, 2 \mathrm{H})$, $6.74(\mathrm{~d}, 2 \mathrm{H}, \mathrm{J}=8.2 \mathrm{~Hz}), 6.69(\mathrm{tt}, 1 \mathrm{H}, \mathrm{J}=8.8,2.3 \mathrm{~Hz}), 3.79$ (bs, 2H). ${ }^{13} \mathrm{C}$ NMR $\left(400 \mathrm{MHz}, \mathrm{CDCl}_{3}\right) \delta$ (ppm) 163.6 (dd, $\mathrm{J}=245.0,13.2 \mathrm{~Hz}), 147.3,144.8(\mathrm{t}, \mathrm{J}=9.6 \mathrm{~Hz}), 129.1$ $(\mathrm{t}, \mathrm{J}=2.5 \mathrm{~Hz}), 128.2,115.6,109.1(\mathrm{dd}, \mathrm{J}=18.4,6.9 \mathrm{~Hz})$, $101.5(\mathrm{t}, \mathrm{J}=25.4 \mathrm{~Hz}) .{ }^{19} \mathrm{~F}$ NMR $\left(376 \mathrm{MHz}, \mathrm{CDCl}_{3}\right) \delta(\mathrm{ppm})$ $-110.8(\mathrm{t}, \mathrm{J}=8.8 \mathrm{~Hz})$. HRMS. $\mathrm{C}_{1} 2 \mathrm{H}_{9} \mathrm{~F}_{2} \mathrm{~N}\left[\mathrm{M}+\mathrm{H}^{+}\right]$Calculated: 206.0781. Found: 206.0780.

3,5-Difluoro-3', 5'-dibromo-4'-aminobiphenyl: ${ }^{34}$ 3,5Difluoro-4'-aminobiphenyl (165.0 $\mathrm{mg}, 0.8 \mathrm{mmol})$ was dissolved in glacial acetic acid $(2.5 \mathrm{ml})$. Bromine $(82 \mu \mathrm{l}$, $255.7 \mathrm{mg}, 1.6 \mathrm{mmol})$ dissolved in glacial acetic acid $(3.8 \mathrm{ml})$ was slowly added. The reaction was left stirring for $1 \mathrm{~h}$. Then water $(5 \mathrm{ml})$ and $\mathrm{CH}_{2} \mathrm{Cl}_{2}(30 \mathrm{ml})$ were added. Sat. $\mathrm{Na}_{2} \mathrm{CO}_{3}$ was added until the reaction mixture became alkaline. The organic phase was washed with sodium thiosulfate $(10 \mathrm{ml})$, water $(10 \mathrm{ml})$, brine $(10 \mathrm{ml})$, and was dried using $\mathrm{MgSO}_{4}$. The organic solvent was removed under reduced pressure affording the title compound as a pale brown solid (271 mg, $93 \%)$. This was used without further purification. ${ }^{1} \mathrm{H}$ NMR (400 MHz, CDCl3) $\delta$ (ppm). 7.59 (s, 2H), 7.01-6.96 (m, 2H), $6.74(\mathrm{tt}, 1 \mathrm{H}, \mathrm{J}=8.84,2.3 \mathrm{~Hz}) 4.69(\mathrm{bs}, 2 \mathrm{H}) .{ }^{13} \mathrm{C} \mathrm{NMR}(400$ $\left.\mathrm{MHz}, \mathrm{CDCl}_{3}\right) \delta(\mathrm{ppm}) 163.6(\mathrm{dd}, \mathrm{J}=246.6,13.1 \mathrm{~Hz}), 130.4$ $(\mathrm{t}, \mathrm{J}=2.6 \mathrm{~Hz}), 130.3,109.29(\mathrm{dd}, \mathrm{J}=18.6,7.2 \mathrm{~Hz}), 109.20$, $102.6(\mathrm{t}, \mathrm{J}=25.2 \mathrm{~Hz}) .{ }^{19} \mathrm{~F} \mathrm{NMR}\left(376 \mathrm{MHz}, \mathrm{CDCl}_{3}\right) \delta(\mathrm{ppm})$ -109.8 (t, J $=8.5 \mathrm{~Hz}$ ). GCMS. $\mathrm{C}_{12} \mathrm{H}_{7} \mathrm{~F}_{2} \mathrm{NBr}_{2}$ Calculated: 362.99 Found: 363 (100), 203 (36), 175 (24), 101 (14).

3,5-difluoro-3',5'-dibromo-4'-cyanobiphenyl: Reaction mixture 1: 3,5-Difluoro-3',5'-dibromo-4'-aminobiphenyl $(270.0 \mathrm{mg}, 0.74 \mathrm{mmol})$ was dissolved in water $(1.0 \mathrm{ml})$ and glacial acetic acid $(3.7 \mathrm{ml})$. Concentrated sulfuric acid $(1.0 \mathrm{ml})$ was added and the reaction mixture was cooled to $10^{\circ} \mathrm{C}$. Sodium nitrite $(56.5 \mathrm{mg}, 0.82 \mathrm{mmol})$ dissolved in a minimum of water was slowly added and the reaction was left stirring for $30 \mathrm{~min}$ at which point, the reaction mixture had become clear. Reaction mixture 2: In a second round, bottom flask $\mathrm{CuSO}_{4} \cdot 5 \mathrm{H}_{2} \mathrm{O}(223 \mathrm{mg}, 0.89 \mathrm{mmol})$ is carefully mixed in water $(1 \mathrm{ml})$ with $\mathrm{KCN}(242 \mathrm{mg}$, $3.72 \mathrm{mmol})$ dissolved in water $(1 \mathrm{ml})$ keeping the temperature below $20{ }^{\circ} \mathrm{C}$ cooling with ice. To reaction mixture 2 is slowly added reaction mixture 1 , while keeping the reaction basic by adding $\mathrm{Na}_{2} \mathrm{CO}_{3}$ (sat.). After the addition is complete, the reaction is left stirring for $1 \mathrm{~h}$ at room temperature. $\mathrm{CH}_{2} \mathrm{Cl}_{2}(30 \mathrm{ml})$ and $\mathrm{Et}_{2} \mathrm{O}(50 \mathrm{ml})$ are added and the organic phase was washed with water $(2 \times 20 \mathrm{ml})$, brine $(20 \mathrm{ml})$, and dried using $\mathrm{MgSO}_{4}$. The solvents were removed under reduced pressure and the crude reaction mixture was purified by column chromatography eluting with pentane: $\mathrm{CH}_{2} \mathrm{Cl}_{2} 3: 1$ to afford the title compound as a colorless solid (141.8 mg, $50 \%) .{ }^{1} \mathrm{H}$ NMR $\left(400 \mathrm{MHz}, \mathrm{CDCl}_{3}\right) \delta(\mathrm{ppm}) 7.8(\mathrm{~s}, 2 \mathrm{H})$, 7.11-7.05 (m, 2H), 6.92 (tt, $1 \mathrm{H}, \mathrm{J}=8.6,2.3 \mathrm{~Hz}) .{ }^{13} \mathrm{C}$ NMR $\left(400 \mathrm{MHz}, \mathrm{CDCl}_{3}\right) \delta(\mathrm{ppm}) 163.7(249.1,12.7 \mathrm{~Hz}), 145.2$, $140.0(\mathrm{t}, \mathrm{J}=9.4 \mathrm{~Hz}), 130.5,127.5,118.4,115.9,110.7$ $(\mathrm{dd}, \mathrm{J}=18.9,7.7 \mathrm{~Hz}), 105.3(\mathrm{t}, \mathrm{J}=25.0 \mathrm{~Hz}) .{ }^{19} \mathrm{~F}$ NMR $\left(376 \mathrm{MHz}, \mathrm{CDCl}_{3}\right) \delta(\mathrm{ppm})-107.7(\mathrm{t}, \mathrm{J}=8.0 \mathrm{~Hz})$. GCMS. $\mathrm{C}_{13} \mathrm{H}_{5} \mathrm{~F}_{2} \mathrm{NBr}_{2}$ Calculated:372.99. Found: 373 (100), 213 (68), 186 (15), 106 (15).

\footnotetext{
${ }^{1}$ A. H. Zewail, Science 242, 1645 (1988).

${ }^{2}$ J. Shao and P. Hänggi, J. Chem. Phys. 107, 9935 (1997)

${ }^{3}$ Y. Fujimura, L. González, K. Hoki, J. Manz, and Y. Ohtsuki, Chem. Phys. Lett. 306, 1 (1999).

${ }^{4}$ D. Gerbasi, M. Shapiro, and P. Brumer, J. Chem. Phys. 115, 5349 (2001).

${ }^{5}$ P. Král and M. Shapiro, Phys. Rev. Lett. 87, 183002 (2001).

${ }^{6}$ K. Hoki, L. González, and Y. Fujimura, J. Chem. Phys. 116, 2433 (2002).

${ }^{7}$ D. Kröner, M. F. Shibl, and L. González, Chem. Phys. Lett. 372, 242 (2003).

${ }^{8}$ D. Kröner and B. Klaumünzer, Chem. Phys. 338, 268 (2007).

${ }^{9}$ E. L. Eliel and A. H. Wilen, in Stereochemistry of Organic Compounds (Wiley, New York, 1994), Chap. 14.

${ }^{10}$ M. Shapiro and P. Brumer, J. Chem. Phys. 95, 8658 (1991).

${ }^{11}$ S. Ramakrishna and T. Seideman, Phys. Rev. Lett. 99, 103001 (2007).

${ }^{12}$ L. H. Coudert, L. F. Pacios, and J. Ortigoso, Phys. Rev. Lett. 107, 113004 (2011).

${ }^{13}$ S. M. Parker, M. A. Ratner, and T. Seideman, J. Chem. Phys. 135, 224301 (2011).

${ }^{14}$ C. B. Madsen, L. B. Madsen, S. S. Viftrup, M. P. Johansson, T. B. Poulsen, L. Holmegaard, V. Kumarappan, K. A. Jørgensen, and H. Stapelfeldt, Phys. Rev. Lett. 102, 073007 (2009)
} 
${ }^{15}$ C. B. Madsen, L. B. Madsen, S. S. Viftrup, M. P. Johansson, T. B. Poulsen, L. Holmegaard, V. Kumarappan, K. A. Jørgensen, and H. Stapelfeldt, J. Chem. Phys. 130, 234310 (2009).

${ }^{16}$ J. J. Larsen, K. Hald, N. Bjerre, H. Stapelfeldt, and T. Seideman, Phys. Rev. Lett. 85, 2470 (2000).

${ }^{17}$ I. Nevo, L. Holmegaard, J. H. Nielsen, J. L. Hansen, H. Stapelfeldt, F. Filsinger, G. Meijer, and J. Küpper, Phys. Chem. Chem. Phys. 11, 9912 (2009).

${ }^{18}$ Dipole moments and polarizabilities were computed with TURBOMOLE 6.2 (Ref. 35) at density functional theory (DFT) level, employing the B3LYP functional which combines Becke's three-parameter hybrid exchange functional ${ }^{36}$ with the Lee-Yang-Parr correlation functional $;{ }^{37}$ the correlation of the uniform electron gas was modeled with the Vosko-WilkNusair VWN5 formulation. ${ }^{38}$ The doubly-polarized triple-zeta basis set, TZVPP, ${ }^{39}$ was used throughout. This level of theory has been shown to be suitable for the study of biphenyls. ${ }^{40}$

${ }^{19}$ L. Holmegaard, J. H. Nielsen, I. Nevo, H. Stapelfeldt, F. Filsinger, J. Küpper, and G. Meijer, Phys. Rev. Lett. 102, 023001 (2009).

${ }^{20}$ F. Filsinger, J. Küpper, G. Meijer, L. Holmegaard, J. H. Nielsen, I. Nevo, J. L. Hansen, and H. Stapelfeldt, J. Chem. Phys. 131, 064309 (2009).

${ }^{21}$ J. L. Hansen, L. Holmegaard, L. Kalhøj, S. L. Kragh, H. Stapelfeldt, F. Filsinger, G. Meijer, J. Küpper, D. Dimitrovski, M. Abu-samha, C. P. J. Martiny, and L. B. Madsen, Phys. Rev. A 83, 023406 (2011).

${ }^{22}$ The spotsizes were determined by scanning a $10 \mu \mathrm{m}$ pinhole across the foci of the laser beams. All peak intensities quoted in the text are calculated using the following formula $I_{0}=\frac{4 \sqrt{\ln 2}}{\pi^{3 / 2}} \frac{{ }_{\text {pulse }}}{\omega_{0}^{2} \tau}$, where $\omega_{0}$ is the spot size measured from the center of the profile till the position where the the intensity has dropped to $I_{0} / e$, and $\tau$ is the pulse duration measured at FWHM.

${ }^{23}$ The dynamic polarizabilities of the molecule, at equilibrium, $\lambda=800 \mathrm{~nm}$ are $\alpha_{z z}=46.83 \AA^{3}, \alpha_{y y}=16.82 \AA^{3}, \alpha_{x x}=32.02 \AA^{3}$.

${ }^{24}$ H. Stapelfeldt and T. Seideman, Rev. Mod. Phys. 75, 543 (2003).

${ }^{25}$ J. L. Hansen, H. Stapelfeldt, D. Dimitrovski, M. Abu-samha, C. P. J. Martiny, and L. B. Madsen, Phys. Rev. Lett. 106, 073001 (2011).

${ }^{26}$ L. J. Frasinski, K. Codling, and P. A. Hatherly, Science 246, 1029 (1989).

${ }^{27}$ J. W. Goodman, in Statistical Optics (Wiley, New York, 1985).

${ }^{28}$ R. Dörner, V. Mergel, O. Jagutzki, L. Spielberger, J. Ullrich, R. Moshammer, and H. Schmidt-Böcking, Phys. Rep. 330, 95 (2000).

${ }^{29}$ J. Ullrich, R. Moshammer, A. Dorn, R. Dörner, L. Schmidt, and H. Schmidt-Böcking, Rep. Prog. Phys. 66, 1463 (2003).

${ }^{30}$ R. H. Brown and R. Q. Twiss, Nature (London) 178, 1046 (1956)

${ }^{31}$ W. P. Aue, E. Bartholdi, and R. R. Ernst, J. Chem. Phys. 64, 2229 (1976).

${ }^{32}$ C. Schröter, K. Kosma, and T. Schultz, Science 333, 1011 (2011).

${ }^{33}$ D. Korolev and N. Bumagin, Tetrahedron Lett. 46, 5751 (2005).

${ }^{34}$ T. Itoh, Y. Nakata, K. Hirai, and H. Tomioka, J. Am. Chem. Soc. 128, 957 (2006).

${ }^{35}$ R. Ahlrichs, M. Bär, M. Häser, H. Horn, and C. Kölmel, Chem. Phys. Lett. 162, 165 (1989).

${ }^{36}$ A. D. Becke, J. Chem. Phys. 98, 5648 (1993).

${ }^{37}$ C. Lee, W. Yang, and R. G. Parr, Phys. Rev. B 37, 785 (1988).

${ }^{38}$ S. H. Vosko, L. Wilk, and M. Nusair, Can. J. Phys. 58, 1200 (1980).

${ }^{39}$ A. Schäfer, C. Huber, and R. Ahlrichs, J. Chem. Phys. 100, 5829 (1994).

${ }^{40}$ M. P. Johansson and J. Olsen, J. Chem. Theory Comput. 4, 1460 (2008). 